\title{
Tuberculosis case finding in first-degree relative contacts not living with index tuberculosis cases in Kampala, Uganda
}

This article was published in the following Dove Press journal:

Clinical Epidemiology

13 October 2015

Number of times this article has been viewed

\author{
Phalkun Chheng ${ }^{1,2}$ \\ Mary Nsereko ${ }^{2}$ \\ LaShaunda L Malone ${ }^{2}$ \\ Brenda Okware ${ }^{2}$ \\ Sarah Zalwango ${ }^{2}$ \\ Moses Joloba ${ }^{2,3}$ \\ W Henry Boom ${ }^{2}$ \\ Ezekiel Mupere ${ }^{1,2,4}$ \\ Catherine M Stein ${ }^{1,2}$
}

\section{On behalf of the \\ Tuberculosis Research Unit}

'Department of Epidemiology and Biostatistics, Case Western Reserve University, Cleveland, OH, USA; ${ }^{2}$ Uganda-Case Western Reserve University Research Collaboration, ${ }^{3}$ Department of Medical Microbiology, College of Health Sciences, Makerere University, Kampala, Uganda; ${ }^{4}$ Department of Pediatrics and Child Health, College of Health Sciences, Makerere University, Kampala, Uganda

Correspondence: Catherine M Stein Department of Epidemiology and Biostatistics, Wolstein Research Building, Room 1316, Case Western Reserve University, Cleveland, OH 44106, USA

$\mathrm{Tel}+|216368563|$

Fax + I 2163684880

Email catherine.stein@case.edu
Purpose: To assess the prevalence of pulmonary tuberculosis among first-degree relative (FDR) contacts not living with tuberculosis (TB) cases.

Methods: A cross-sectional analysis of household contacts living with an index TB case and FDR contacts living outside of households in Kampala, Uganda, is presented.

Results: A total of 177 contacts (52 FDRs and 125 index household contacts) of 31 TB cases were examined. Compared with index household contacts, FDR contacts were older, more likely to be TB symptomatic ( $50 \%$ vs $33 \%$ ), had a higher percentage of abnormal chest X-rays (19\% vs $11 \%)$, sputum smear positive (15\% vs $5 \%$ ), and many similar epidemiologic risk factors, including HIV infection (13\% vs 10\%). Contact groups had similar pulmonary tuberculosis prevalence: $9.6 \%$ in FDR vs $10.4 \%$ in index household contacts and similar Mycobacterium tuberculosis infection: $62 \%$ in FDR vs $61 \%$ in index households.

Conclusion: TB is common among FDR contacts. High TB prevalence justifies targeting FDRs during household contact investigations. Combining TB active-case finding among FDR contacts with household contact investigation in low-income setting is feasible. This should be part of national TB control program strategies for increasing TB case-detection rates and reducing community TB transmission and death.

Keywords: prevalence of pulmonary tuberculosis, limited resource setting, contact tracing

\section{Introduction}

Tuberculosis (TB) remains one of the leading causes of mortality and morbidity worldwide. The World Health Organization (WHO) estimates that approximately one-third of the world population is infected with Mycobacterium tuberculosis (Mtb), the agent that causes Mtb infection and disease, and annually eight million individuals develop TB disease, accounting for at least two million deaths. ${ }^{1}$ In Uganda, one of 22 high-burden countries with TB cases, WHO estimated 62,000 TB incident cases and that in 2013, 20,648 of them were also coinfected with HIV.

Current TB control strategies have limited success in identifying all infectious TB source cases and their contacts in the community, especially in developing countries, ${ }^{2,3}$ such as Uganda, one of the world's highest TB burden countries. Contact investigation among TB patients is a systematic evaluation to identify active disease or latent Mtb infection (LTBI) among contacts of known TB patients. While it is a priority in highincome countries, human and financial resource constraints in many high-burden countries make contact investigation a low priority in the national TB control strategy. WHO has recommended two especially high-priority groups, children aged under 5 years and people infected with human immunodeficiency virus (HIV), who are at 
highest risk of progression to TB disease following contact with a TB patient in a household setting. ${ }^{4-6}$ Household contact investigations are considered as an important approach among other active-case finding strategies to increase TB case-detection rates and to interrupt the transmission of TB disease. ${ }^{3,7-11}$ Household contacts are at high risk of Mtb infections $^{12-15}$ and developing active TB. ${ }^{16-19}$

Previous studies have shown that TB transmission is associated with intimacy or proximity and time spent with the index TB case. ${ }^{13,20-22}$ Several other studies have demonstrated a role for host genetics in susceptibility to LTBI and TB disease. ${ }^{23,24}$ While the risk of getting Mtb infection or active disease was determined for the first-degree relatives (FDRs) who are mostly close contacts being targeted in household contact investigation, ${ }^{13,25-27}$ no studies have examined this same risk for other first-degree relatives living in separate households who may also be, to some extent, exposed through interaction with index TB cases.

The purpose of this study was to assess the outcome of case finding among FDR contacts living outside the home of culture-confirmed index TB cases and to compare clinical characteristics of active TB among FDR contacts with that of index household (IHH) contacts who are exposed to the same index TB cases.

\section{Materials and methods Study design and data collection}

We conducted a cross-sectional study analyzing data of the index TB cases, their IHH contacts living in the same household, and FDR contacts living in a separate household who were enrolled in a prospective cohort study called the Kawempe Community Health Study between April 2002 and December 2007 in a TB Research Clinic in Kampala, Uganda. ${ }^{13,28}$

After obtaining written informed consent, the study recruited eligible index cases who had sputum culture-positive pulmonary TB, aged 18 or more years, and who had at least one household contact living with them. Home health visitors enumerated household contacts and then FDR contacts living outside the home of each index case on census forms.

As part of the index household evaluation, IHH contacts, including first-degree relatives (parents, children, and siblings), living in the index home were contacted by home health visitors who made an initial home visit. Performed within 2 weeks of confirmed TB diagnosis for index cases, the purpose of the initial visit was to inform household contacts about the study, provide health education about TB, and obtain written informed consent from all adult household members and parents or guardians for children $<18$ years.
Home health visitors followed the same procedure when they visited and enrolled FDR contacts living in a separate household. Due to logistical constraints, visits to FDRs households were restricted to travel within a $50 \mathrm{~km}$ radius ( $\sim 2$ hours of travel) from the study clinic. As part of FDR household evaluations, parents, children, and siblings of the index TB cases were evaluated. Later on, due to limited staffing and other logistical issues, only FDR households with FDRs who were TB suspects were evaluated. This change in approach occurred only after three FDR households were enrolled that were beyond $50 \mathrm{~km}$, so this number was too small to perform stratified analyses. Through health education about TB, the index cases were encouraged to invite FDRs with symptoms suggestive of TB to the study clinic for evaluation. On presentation to the clinic, these suspected cases underwent a full evaluation. Throughout this paper, the term "FDR contacts" refers to FDRs living outside the index household. These FDR contacts did not live with any other index cases who were enrolled in our study.

Once a household was identified (either IHH or FDR), all individuals within that household providing informed consent were clinically characterized.

\section{Case definitions}

An index TB case was defined as the first culture-confirmed TB patient who already had at least two acid-fast bacilli (AFB)positive smears and was referred from a hospital of the national TB program. An IHH contact was defined as an individual who had resided in the index household for at least 7 consecutive days during the 3 months period prior to the diagnosis of TB in the index case, while FDR contacts were parents, siblings or offspring of the index case, who did not reside in the household of the index case. Coprevalent TB cases were contacts with active TB disease who were diagnosed with active TB (definite or probable $)^{29}$ within 60 days from the day of TB diagnosis in the index case, and incident case were those diagnosed with active TB after 60 days from the diagnosis of TB in index case.

\section{Measurements}

Both contact groups were assessed for the risk of Mtb infection and disease in a similar way by home health visitors. During each household visit, home health visitors conducted an assessment of individual TB risk factors for all recruited IHH and FDR contacts, using a standard questionnaire that included information such as demographics, occupation, medical history, degree of contact with the index case, signs and symptoms, vital signs, and anthropometric measurements. To assess LTBI, home health visitors performed a tuberculin skin test (TST), 
with purified protein derivative using the Mantoux method and returned between 48 hours and 72 hours to measure the diameter of induration in millimeters using the "ball-point" technique. TST positive was defined as induration $\geq 10 \mathrm{~mm}$ in all contacts and induration $\geq 5 \mathrm{~mm}$ for 5 years old or younger children and HIV-infected patients. LTBI was defined as those with skin test positive and TB disease was ruled out. Vaccination with Bacille Calmette-Guérin (BCG) was determined by the presence of a characteristic scar on the left deltoid and was verified by the use of available medical records. Any contact suspected of having active TB underwent formal TB evaluation, including chest radiography, sputum smear and culture examinations, and TB drug susceptibility, if indicated. HIV status was determined by the enzyme-linked immunosorbent assay (ELISA) method using commercial diagnostic kits (Cambridge BioScience, Worcester, MA, USA). Mtb lineage in the index case was examined as previously described, ${ }^{30}$ by genotyping eight single nucleotide polymorphisms in DNA extracted from stored Mtb isolates, then grouping based on the phylogenetic analysis of Gagneux and Small. ${ }^{31}$

\section{Statistical analysis}

The main objective of this analysis was to assess the yield of TB case finding among FDR contacts compared to that of IHH contacts of the same index TB cases and to compare characteristics associated with TB among FDR and IHH contacts. The main outcome was active TB among FDR and IHH contacts. First, index TB cases were analyzed in two groups to evaluate comparability between 1) index cases with an FDR evaluated and 2) index cases who did not have an FDR evaluated, to determine if any significant differences exist between the households where the FDRs were evaluated. Note that this analysis does not compare "included" versus "excluded" index cases. Univariate analysis of continuous variables included calculation of the median (with interquartile range [IQR]). Categorical variables were reported as frequencies. In bivariate analysis, continuous variables were compared using Student's unpaired $t$-test or Mann-Whitney-Wilcoxon test for unpaired data, and for categorical variables, proportions were compared using chi-square test or Fisher's exact test, where appropriate. A $P$-value of $<0.05$ was considered statistically significant. SAS version 9.3 was used in these analyses.

\section{Ethical review}

The study was approved by University Hospitals Institutional Review Board at University Hospitals Case Medical Center in the United States (IRB number 10-01-25) and the National

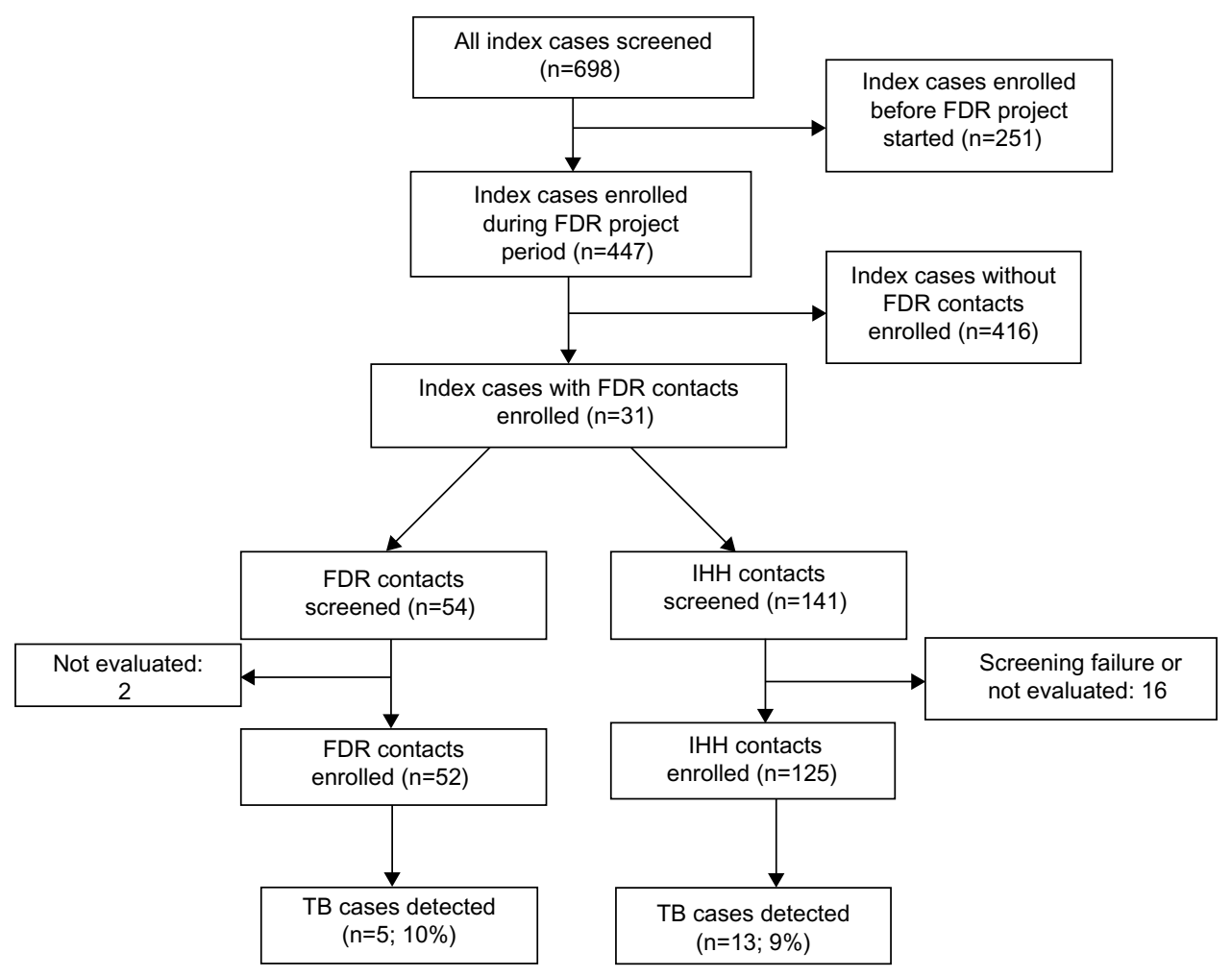

Figure I Study algorithm.

Abbreviations: FDR, first-degree relative; $\mathrm{IHH}$, index household; $\mathrm{TB}$, tuberculosis. 
HIV/AIDS Research Committee and Uganda National Council for Science and Technology in Uganda (reference number MV 658).

\section{Results \\ Index TB cases}

Among 447 highly infectious TB index cases with advanced, bacteriologically culture-confirmed TB disease who were enrolled in the Kawempe Community Health Study from April 2002 to December 2007, only 31 index cases had FDR contacts evaluated and enrolled in this study (Figure 1). To assess potential differences between index TB cases with FDR contacts evaluated and those without FDR contacts enrolled, we compared household outcomes of the 31 index TB cases who had FDRs evaluated with the 416 index TB cases without FDR contacts enrolled (those with only IHH contacts enrolled). HIV infection rates were very high (42\%) among both index groups. There was no significant difference between characteristics of the two index TB case groups, except the prevalence of TST positivity at baseline and TST conversion among IHH contacts of index group without FDR contacts enrolled was higher (74\% vs 65\%), as well as hemoptysis (20\% vs $10 \%)$ as shown in Table 1.

\section{FDR and IHH contacts}

A total of 177 contacts (52 FDR and 125 IHH contacts) of 31 index cases were included in this analysis (Figure 1). Compared with IHH contacts, FDR contacts were older (median age 28 [IQR $=13-48]$ vs 11 [5-21]), more likely to be TB symptomatic ( $50 \%$ vs 33\%), had higher rates of abnormal chest x-ray (CXR) (19\% vs $11 \%$ ), and sputum smear positive (15\% vs 5\%). FDR contacts still had many of the epidemiologic risk factors, such as sleeping in the same room, sharing meals, some frequent contact with index case, but at lower proportion compared with those of IHH contacts (Table 2). Even though these rates are significantly lower as compared with those of IHH contacts, which we would expect by design, FDR contacts still were at risk of exposure to associated household index TB cases. Both contact groups reported having contact with other TB cases ( $15 \%$ for FDR vs $22 \%$ for IHH contacts) as well. Finally, HIV infection, one of the main risk factors for Mtb infection and reactivation of active $\mathrm{TB}$, was approximately the same rate in both contact groups (13\% vs 10\%).

\section{Outcome of investigation at baseline and follow-up}

Among the 177 contacts of the index group with FDR contacts enrolled, 18 active TB cases including five (9.6\%)
Table I Demographics and clinical characteristics of index TB cases

\begin{tabular}{|c|c|c|}
\hline \multirow[t]{2}{*}{ Characteristic } & $\begin{array}{l}\text { Index case/s } \\
\text { with FDR } \\
(\mathbf{N}=3 \text { I) }\end{array}$ & $\begin{array}{l}\text { Index case/s } \\
\text { without FDR } \\
(\mathrm{N}=4 \mid 6)\end{array}$ \\
\hline & n (\%) & $n(\%)^{a}$ \\
\hline \multicolumn{3}{|l|}{ Age (years) } \\
\hline Median (IQR) & $32(25-36)$ & $27(23-35)$ \\
\hline Sex, male & $20(65)$ & $2 I I(5 I)$ \\
\hline \multicolumn{3}{|l|}{ BMI $\left(\mathrm{kg} / \mathrm{m}^{2}\right)$} \\
\hline Median (IQR) & $19(17-20)$ & $19(17-20)$ \\
\hline Cough vs none & $31(100)$ & $402(97)$ \\
\hline Hemoptysis vs none & $3(10)$ & $63(20)$ \\
\hline BCG scars vs none & $17(55)$ & $24 I(58)$ \\
\hline \multicolumn{3}{|l|}{ TST positive (baseline) } \\
\hline Yes & $25(8 I)$ & $357(87)$ \\
\hline No & $6(19)$ & $55(13)$ \\
\hline \multicolumn{3}{|l|}{ Cavitary disease } \\
\hline Yes & $15(48)$ & $250(60)$ \\
\hline No & $16(52)$ & $156(38)$ \\
\hline \multicolumn{3}{|l|}{$\mathrm{AFB}+$ smear } \\
\hline Yes & $29(94)$ & $407(98)$ \\
\hline No & $2(6)$ & $9(2)$ \\
\hline Culture converted after & $18(86)$ & $230(79)$ \\
\hline \multicolumn{3}{|l|}{2 months of TB treatment } \\
\hline \multicolumn{3}{|l|}{ Mtb strains } \\
\hline Euro-American Ugandan-I & $13(48)$ & 191 (49) \\
\hline Euro-American non-Ugandan & $7(26)$ & $87(22)$ \\
\hline Central Asian & $3(I I)$ & $39(10)$ \\
\hline Euro-American Ugandan-2 & $3(\mathrm{II})$ & $55(14)$ \\
\hline Other & I (4) & $7(2)$ \\
\hline \multicolumn{3}{|l|}{ HIV test } \\
\hline Positive & $13(42)$ & $174(42)$ \\
\hline Negative & $18(58)$ & $24 \mid(58)$ \\
\hline \multicolumn{3}{|l|}{ Treatment failure } \\
\hline Yes & $\mathrm{I}(3)$ & $10(2)$ \\
\hline No & $30(97)$ & $406(98)$ \\
\hline \multicolumn{3}{|l|}{ Relapse } \\
\hline Yes & $3(10)$ & $35(8)$ \\
\hline No & $28(90)$ & $381(92)$ \\
\hline \multicolumn{3}{|l|}{ Total enrolled IHH outcomes ${ }^{\mathrm{b}}$} \\
\hline Coprevalent + incident cases ${ }^{c}$ & $13(10)$ & $110(8)$ \\
\hline TST positive at baseline & $69(60)$ & $862(68)$ \\
\hline TST converter & $12(10)$ & $145(11)$ \\
\hline TST positive at baseline + converter & $81(65)$ & $1007(74)$ \\
\hline
\end{tabular}

Notes: aFrequencies do not always add to the total number because of missing values; 'total index household $(\mathrm{IHH})$ contacts enrolled during the same FDR project period $=1484$; 'total number of household contacts who become coprevalent and or incident cases. All TB cases were clinically classified as definite or probable based on ATS criteria. ${ }^{29}$

Abbreviations: TB, tuberculosis; FDR, first-degree relative; IQR, interquartile range; BMI, body mass index; TST, tuberculin skin test; HIV, human immunodeficiency virus; IHH, index household; BCG, Bacille Calmette-Guerin; AFB, acid-fast bacilli; ATS, American Thoracic Society.

among FDR contacts and 13 (10.4\%) among IHH contacts were detected. Both contact groups had similar rates of $\mathrm{Mtb}$ infection (baseline-positive TST of $62 \%$ vs $61 \%$ ). FDR cases were older, had higher proportion of males (60\% vs $38 \%)$, 
Table 2 Demographics and clinical characteristics of FDR and $\mathrm{IHH}$ contacts

\begin{tabular}{|c|c|c|c|}
\hline \multirow[t]{2}{*}{ Characteristic } & $\begin{array}{l}\text { FDR contacts } \\
(\mathrm{N}=52)^{\mathrm{a}}\end{array}$ & $\begin{array}{l}\text { IHH contacts } \\
(\mathrm{N}=\mid 25)^{\mathrm{a}}\end{array}$ & \multirow[t]{2}{*}{ OR (95\% Cl) } \\
\hline & $\%$ & $\%$ & \\
\hline
\end{tabular}

\begin{tabular}{|c|c|c|c|c|c|}
\hline \multicolumn{6}{|l|}{ Age (years) } \\
\hline Median (IQR) & 28 & $13-48$ & 11 & $5-21$ & $14(6-28)^{b}$ \\
\hline \multicolumn{6}{|c|}{ Age distribution (years) } \\
\hline $0-4$ & 5 & 10 & 31 & 25 & \multirow[t]{6}{*}{$\mathrm{NA}^{\mathrm{c}}$} \\
\hline $5-14$ & 11 & 22 & 44 & 35 & \\
\hline $15-24$ & 8 & 16 & 21 & 17 & \\
\hline $25-34$ & 7 & 14 & 16 & 13 & \\
\hline $35-44$ & 6 & 12 & 7 & 6 & \\
\hline$\geq 45$ & 15 & 28 & 6 & 5 & \\
\hline \multicolumn{6}{|l|}{ Sex } \\
\hline Female & 31 & 60 & 71 & 57 & \multirow[b]{2}{*}{$0.9(0.5-1.7)$} \\
\hline Male & 21 & 40 & 54 & 43 & \\
\hline \multicolumn{6}{|c|}{ Relationship to index case } \\
\hline Spouse & 0 & 0 & 11 & 9 & \multirow{5}{*}{$10.7(3.6-3 \mid .1)$} \\
\hline Parents & 16 & 31 & 5 & 4 & \\
\hline Children & 17 & 33 & 44 & 35 & \\
\hline Sibling & 14 & 27 & 12 & 10 & \\
\hline Other relatives & 5 & 9 & 53 & 42 & \\
\hline \multicolumn{6}{|c|}{ Frequent contact with the index case } \\
\hline Daily & 7 & 14 & 110 & 88 & \multirow[t]{5}{*}{$0.02(0.01-0.05)$} \\
\hline I-6 days/week & 12 & 23 & 6 & 5 & \\
\hline$<$ I day/week & 11 & 22 & 8 & 6 & \\
\hline None & 18 & 35 & I & 1 & \\
\hline Do not recall & 3 & 6 & 0 & 0 & \\
\hline \multicolumn{6}{|c|}{ Contact time with the index case } \\
\hline Just a short time & 11 & 22 & 10 & 8 & \multirow[t]{4}{*}{$3.5(1.4-8.8)$} \\
\hline Part of the day & 10 & 20 & 63 & 50 & \\
\hline Most of the day & 10 & 20 & 50 & 40 & \\
\hline Do not recall & 4 & 8 & 2 & 2 & \\
\hline \multicolumn{6}{|c|}{ Having meals with index } \\
\hline Yes & 19 & 37 & 123 & 98 & \multirow[t]{3}{*}{$0.02(0-0.1)$} \\
\hline No & 16 & 31 & 2 & 2 & \\
\hline Not applicable & 16 & 31 & 0 & 0 & \\
\hline \multicolumn{6}{|c|}{ Sleep same room with the index case } \\
\hline Yes & 6 & 12 & 68 & 54 & \multirow[t]{3}{*}{$0.2(0.1-0.4)$} \\
\hline No & 29 & 57 & 57 & 46 & \\
\hline Not applicable & 16 & 31 & 0 & 0 & \\
\hline \multicolumn{6}{|c|}{ Share bed with index case } \\
\hline Yes & 0 & 0 & 14 & 11 & \multirow[t]{2}{*}{$0(0-0.8)$} \\
\hline No & 35 & 100 & 110 & 89 & \\
\hline \multicolumn{6}{|c|}{ Contact w/other TB cases } \\
\hline Yes & 8 & 15 & 28 & 22 & \multirow[t]{2}{*}{$0.6(0.3-1.5)$} \\
\hline No & 44 & 84 & 97 & 78 & \\
\hline TB in the past & & & & & \\
\hline Yes & 1 & 2 & 3 & 2 & $0.6(0.1-6.3)$ \\
\hline No & 50 & 98 & 122 & 98 & \\
\hline Any TB symptom & 26 & 50 & 41 & 33 & $2(1.1-3.9)$ \\
\hline Cough & 19 & 37 & 24 & 19 & $2.4(1.2-4.9)$ \\
\hline Hemoptysis & 1 & 2 & 1 & 1 & $2.4(0-192.4)$ \\
\hline Fever & 11 & 22 & 9 & 7 & $3.5(1.3-8.9)$ \\
\hline Weight loss & 12 & 23 & 16 & 13 & $2(0.9-4.7)$ \\
\hline Night sweats & 9 & 18 & 14 & 11 & $1.7(0.7-4.1)$ \\
\hline BMI $\left(\mathrm{kg} / \mathrm{m}^{2}\right)$ & & & & & \\
\hline Median (IQR) & 21 & $17-25$ & 17 & $|6-2|$ & $17(|6-2|)^{b}$ \\
\hline
\end{tabular}

(Continued)
Table 2 (Continued)

\begin{tabular}{|c|c|c|c|c|c|}
\hline \multirow[t]{2}{*}{ Characteristic } & \multicolumn{2}{|c|}{$\begin{array}{l}\text { FDR contacts } \\
(\mathrm{N}=52)^{\mathrm{a}}\end{array}$} & \multicolumn{2}{|c|}{$\begin{array}{l}\text { IHH contacts } \\
(\mathrm{N}=\mid 25)^{\mathrm{a}}\end{array}$} & \multirow[t]{2}{*}{ OR $(95 \% \mathrm{CI})$} \\
\hline & $\mathbf{n}$ & $\%$ & $\mathbf{n}$ & $\%$ & \\
\hline \multicolumn{6}{|c|}{ Extent of disease on CXR } \\
\hline Abnormal & 7 & 19 & 13 & 11 & $1.9(0.6-5.9)$ \\
\hline Normal & 30 & 81 & 110 & 88 & \\
\hline \multicolumn{6}{|c|}{ Baseline AFB + smear } \\
\hline Yes & 4 & 15 & 3 & 5 & $3.5(0.5-25.9)$ \\
\hline No & 22 & 85 & 58 & 94 & \\
\hline \multicolumn{6}{|c|}{ Baseline sputum culture results } \\
\hline Positive & 6 & 23 & 17 & 27 & $0.8(0.3-2.3)$ \\
\hline Negative & 20 & 77 & 44 & 71 & \\
\hline \multicolumn{6}{|l|}{ BCG scar } \\
\hline Yes & 34 & 67 & 79 & 73 & I.I (0.5-2.5) \\
\hline No & 11 & 22 & 29 & 27 & \\
\hline Uncertain & 6 & 12 & 17 & 14 & \\
\hline \multicolumn{6}{|c|}{ Baseline TST positive } \\
\hline Yes & 32 & 62 & 76 & 61 & I (0.5-2) \\
\hline No & 18 & 35 & 49 & 39 & \\
\hline \multicolumn{6}{|l|}{ HIV status } \\
\hline Positive & 7 & 13 & 13 & 10 & I.4 (0.5-3.7) \\
\hline Negative & 42 & 81 & 109 & 87 & \\
\hline Unknown & 3 & 6 & 3 & 3 & \\
\hline TB cases detected ${ }^{d}$ & 5 & 9.6 & 13 & 10.4 & \\
\hline Yes & 5 & 9.6 & 13 & 10.4 & $0.9(0.3-2.7)$ \\
\hline No & 47 & 90.4 & 112 & 89.6 & \\
\hline
\end{tabular}

Notes: ${ }^{\mathrm{a} F}$ requencies do not always add to the total number because of missing values; bvalues are median (interquartile range); 'OR not computed because there tended to not be children in the FDR households based on the study design; dall TB cases were clinically classified as definite or probable based on ATS criteria. ${ }^{29}$

Abbreviations: $F D R$, first-degree relative; $I H H$, index household; OR, odds ratio; $\mathrm{Cl}$, confidence interval; IQR, interquartile range; $\mathrm{NA}$, not applicable; $\mathrm{TB}$, tuberculosis; CXR, chest X-ray;AFB, acid-fast bacilli; BCG, Bacillus Calmette-Guérin;TST, tuberculin skin test; HIV, human immunodeficiency virus; ATS, American Thoracic Society.

cough ( $80 \%$ vs $46 \%$ ), extent of disease on CXR (abnormal vs other) ( $80 \%$ vs $38 \%$ ), positive AFB smear ( $60 \%$ vs $27 \%$ ) and negative sputum culture ( $40 \%$ vs $9 \%$ ), and more likely to have cavitary disease on CXR (Table 3). Among the five FDR TB cases, four of them were TB symptomatic, and they presented with cough for at least 1 month, had moderate-toadvanced disease on CXR, positive sputum smear, no TB in the past, but three of them also had history of contacts with other TB cases besides the index case. All of them were HIV negative (Table 4). Though none of these comparisons achieved statistical significance because of small sample size, there were many clinically meaningful differences between FDR and IHH TB cases. FDR cases were more likely to be male, cough, have an abnormal chest X-ray, be AFB positive, and were less likely to have hemoptysis.

Finally, we conducted subgroup analyses (data not shown) to assess the robustness of our findings. First, we examined whether daily contact with the index case significantly influenced the risk of TB in FDR versus IHH contacts. Only one FDR TB case $(20 \%)$ had daily contact with the index 
Table 3 Characteristics of TB cases detected among FDR and $\mathrm{IHH}$ contacts

\begin{tabular}{|c|c|c|c|c|c|}
\hline \multirow[t]{2}{*}{ Characteristic } & \multicolumn{2}{|c|}{$\begin{array}{l}\text { FDR TB } \\
\operatorname{cases}(N=5)^{a}\end{array}$} & \multicolumn{2}{|c|}{$\begin{array}{l}\text { IHH TB } \\
\text { cases }(\mathrm{N}=13)^{\mathrm{a}}\end{array}$} & \multirow[t]{2}{*}{ OR $(95 \% \mathrm{Cl})$} \\
\hline & $\mathbf{n}$ & $\%$ & $\mathbf{n}$ & $\%$ & \\
\hline \multicolumn{6}{|l|}{ Age (years) } \\
\hline Median (IQR) & 40 & $17-45$ & 6 & $2-8$ & $7(3-25)^{b}$ \\
\hline \multicolumn{6}{|c|}{ Age groups (years) } \\
\hline$<15$ & I & 20 & 10 & 77 & \\
\hline$\geq 15$ & 4 & 80 & 3 & 23 & | $3.3(0.7-7 \mid 9.9)$ \\
\hline \multicolumn{6}{|l|}{ Sex } \\
\hline Male & 3 & 60 & 5 & 38 & $2.4(0.2-36.8)$ \\
\hline Female & 2 & 40 & 8 & 62 & \\
\hline \multicolumn{6}{|l|}{ Cough } \\
\hline Yes & 4 & 80 & 6 & 46 & $4.7(0.3-26 \mid .9)$ \\
\hline No & I & 20 & 7 & 54 & \\
\hline \multicolumn{6}{|l|}{ BCG scars } \\
\hline Present & I & 25 & 7 & 54 & $0.2(0-6.4)$ \\
\hline Absent & I & 25 & 3 & 23 & \\
\hline Uncertain & 2 & 50 & 3 & 23 & \\
\hline \multicolumn{6}{|l|}{ TST status } \\
\hline Positive & 3 & 60 & 7 & 54 & I.3 (0.I-20.I) \\
\hline Negative & 2 & 40 & 6 & 46 & \\
\hline \multicolumn{6}{|c|}{ Extent of disease on CXR } \\
\hline Abnormal & 4 & 80 & 5 & 38 & $6.4(0.4-353.6)$ \\
\hline Normal & I & 20 & 8 & 62 & \\
\hline \multicolumn{6}{|c|}{ Cavitary disease on CXR } \\
\hline Yes & 2 & 40 & 0 & 0 & Not estimable \\
\hline No & 3 & 60 & 13 & 100 & \\
\hline \multicolumn{6}{|l|}{ AFB smear } \\
\hline Positive & 3 & 60 & 3 & 27 & $4(0.3-66.1)$ \\
\hline Negative & 2 & 40 & 8 & 73 & \\
\hline \multicolumn{6}{|l|}{ Sputum culture } \\
\hline Positive & 3 & 60 & 10 & 91 & $0.5(0-4.4)$ \\
\hline Negative & $2^{c}$ & 40 & $I^{c}$ & 9 & \\
\hline \multicolumn{6}{|l|}{ HIV status } \\
\hline Positive & 0 & 0 & 2 & 15 & $0(0-9.3)$ \\
\hline Negative & 5 & 100 & 11 & 85 & \\
\hline
\end{tabular}

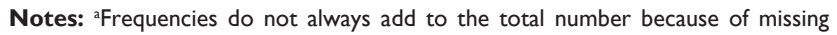
values; 'values are median (interquartile range); ' these TB cases were clinically active TB with $C X R$ consistent with TB and improved after receiving TB treatment.

Abbreviations: TB, tuberculosis; FDR, first-degree relative; $I \mathrm{HH}$, index household; $\mathrm{OR}$, odds ratio; $\mathrm{Cl}$, confidence interval; $\mathrm{IQR}$, interquartile range; $\mathrm{BCG}$, Bacillus Calmette-Guérin; TST, tuberculin skin test; CXR, chest X-ray; AFB, acid-fast bacilli; HIV, human immunodeficiency virus.

case, versus eleven (84\%) of IHH contacts diagnosed with TB. This is actually the opposite of what one would expect and suggests that the frequency of contact was not the primary determinant of TB incidence in FDR households. Second, we restricted the analyses among the IHH contacts to those with age $\geq 15$ years, since children of the index case are unlikely to live outside their home (eg, in an FDR household), thus making the two groups more comparable in age. Among IHH contacts who were of age $\geq 15$ years, the presence of most of the variables summarized in Table 2 were similar, except the prevalence of TST positivity was higher (76\%). The major difference was that only three TB cases were diagnosed in IHH contacts ( $6 \%$ of those age $\geq 15$ years), compared to four cases in FDR contacts age $\geq 15$ years $(11 \%)$; because of the small sample sizes here, no statistical analyses are presented, but it is still interesting to note that the prevalence of TB in FDR contacts was almost double that in IHH contacts. FDR TB cases also were similar to the IHH adult TB cases with respect to the prevalence of TB symptoms, including cough, fever, hemoptysis, weight loss, and night sweat. However, FDR TB cases were more severe, with higher proportion of extent of disease on CXR ( $75 \%$ vs $33 \%$ ), cavitary disease (50\% vs none), and higher sputum smear positivity rate ( $75 \%$ vs $33 \%$ ). However, these observations are made with caution because of the small sample size.

\section{Discussion}

Our study demonstrated a relatively high yield of active TB cases resulting from contact investigation among both IHH and FDR contacts to culture-confirmed TB cases. We documented a high prevalence of TB cases (10\%) among both IHH and FDR contacts of the same index patients with TB. High prevalence of active TB among FDR contacts could be explained by the nature of our study design by selecting and evaluating only FDR contacts of index case households where one or more individuals had potential TB symptoms. While logistical issues resulted in the medical team prioritizing certain households for evaluation, thus resulting in a potentially inflated prevalence of TB within these FDR households, this actually demonstrates the efficiency of identifying TB cases using a targeted and focused approach. Another explanation for this could be that even though they were living in a separate household, these FDR contacts who were immediate family members of the index case also had more or less the same contact or exposure with their index case as IHH contacts, as they might go and visit more often or stay with the index case for certain periods of time to provide care for the index cases who were sick, and this is the traditional practice or culture in this African country. Tracing of FDRs was limited to within $50 \mathrm{~km}$ of a large urban center, Kampala, Uganda. As such, higher TB prevalence observed in FDR contacts may be affected by proximity to a large urban center, whereas FDRs living in more rural areas may have a lower prevalence of TB both because of less exposure to the index case and lower population density. ${ }^{32-34}$ Finally, this could be explained by a role of genetic factors in susceptibility to LTBI and TB disease. ${ }^{23,24}$ The TB case-detection rate of $10 \%$ among IHH contacts was higher compared to other reports (from $<1 \%$ to $7.8 \%$ ) by 
Table 4 Summary of FDR contacts diagnosed with TB

\begin{tabular}{|c|c|c|c|c|c|c|c|c|c|c|}
\hline Sex & $\begin{array}{l}\text { Age } \\
\text { (years) }\end{array}$ & $\begin{array}{l}\text { Any TB } \\
\text { symptom }\end{array}$ & $\begin{array}{l}\text { Cough } \\
\text { duration (days) }\end{array}$ & $\begin{array}{l}\text { CXR extent } \\
\text { of disease }\end{array}$ & $\begin{array}{l}\text { CXR cavitary } \\
\text { disease }\end{array}$ & $\begin{array}{l}\text { Sputum } \\
\text { smear }\end{array}$ & $\begin{array}{l}\text { Sputum } \\
\text { culture }\end{array}$ & $\begin{array}{l}\text { Contact with } \\
\text { other TB cases }\end{array}$ & $\begin{array}{l}\text { TB in } \\
\text { the past }\end{array}$ & $\begin{array}{l}\text { HIV } \\
\text { result }\end{array}$ \\
\hline Male & 40 & Yes & 30 & Advanced & Yes & Positive & Positive & Yes & No & Negative \\
\hline Female & 64 & Yes & 28 & Advanced & No & Positive & Negative & No & No & Negative \\
\hline Male $^{\mathrm{a}}$ & 4 & Yes & 24 & Moderate & No & Negative & Negative & Yes & No & Negative \\
\hline Male & 17 & Yes & 84 & Normal & No & Positive & Positive & Yes & No & Negative \\
\hline Female & 45 & Yes & 30 & Moderate & Yes & Negative & Positive & No & No & Negative \\
\hline
\end{tabular}

Note: a This child was classified as "probable TB." 43

Abbreviations: FDR, first-degree relative; TB, tuberculosis; CXR, chest X-ray; HIV, human immunodeficiency virus.

previous studies. ${ }^{10,11,13,26,35}$ Unfortunately, we were not able to compare this TB case-detection rate among FDR contacts with findings by other studies as there has been no data or assessment on these particular FDR contacts who live separately from index TB cases. However, this proportion is also very high even compared with that of IHH contacts from other studies. ${ }^{10,11,13,26,35}$ This high prevalence of TB could be due to the fact that these contacts were exposed to more highly infectious, symptomatic, sputum smear positive, and culture-confirmed pulmonary TB cases. However, this is not clearly supported by our data: on the one hand, index cases without FDRs actually had higher proportion of Mtb infection as evidenced by the higher proportion of TST positivity, but on the other hand, index cases without FDRs had greater severity of disease on CXR (Table 1). Furthermore, most of the TB cases occurring within IHH households were in children (10 out of 13) who are more susceptible to TB.

Our findings suggest that FDR contacts are among high risk groups of TB and thus should be targeted in household or any contact investigation to efficiently screen for TB to improve TB case detection. Furthermore, the clinical characteristics seen in the FDR TB cases suggest that their disease might be more advanced. This intervention, which supports recent WHO recommendations for investigating contacts of persons with infectious TB in low- and middle-income countries, ${ }^{6}$ could detect potentially more TB cases who were previously undiagnosed through household contact investigations or routine TB programs that do not target this contact group. ${ }^{36}$ Additionally, this is likely the first study that assesses the risk of TB among FDR contacts who live separately from index TB cases. Previous studies of active TB case finding in Kampala, Uganda, revealed the prevalence of TB in the community of $0.76 \%$ and $3.5 \%$, with the higher proportion being identified in the slums. ${ }^{37,38}$ Those studies searched door-to-door for chronic coughers, then checked for TB in those individuals. In this present study, by focusing on FDR contacts with TB symptoms, the yield of TB case finding was higher.
More active TB cases can be identified through different strategies of active-case finding (ACF), including contact investigation, in which the yield is varied by design and program implementation. ${ }^{39}$ Since there have been many different contact tracing strategies and for logistical reasons, Fox et al cautioned against utilizing these interventions and also suggested that additional strategies that enhance case finding should be cost-effective. ${ }^{35}$ Nishikiori et al also suggested the importance of appropriate target populations and careful selection of cost-effective diagnostic strategies for $\mathrm{ACF}{ }^{40}$ Yadav et al found that ACF programs targeting household and neighborhood contacts in Cambodia was highly cost-effective and stressed the importance of selecting the right target of the program, especially among symptomatic household and neighborhood contacts. ${ }^{41}$ Moreover, Sekandi et al found that adding household contact investigations to existing passive case finding programs was more costeffective than combining it to community $\mathrm{ACF}^{42}$

However, our study was subject to several limitations. As described earlier, due to the nature of our study design and other logistical issues we were not able to screen more FDR contacts and this selection most likely resulted in selection of more symptomatic patients. Because potential FDR TB cases were initially identified through report by the index case, there is a strong possibility of underreporting of TB cases. Furthermore, it is likely that these FDR contacts had greater contact with the index cases. Thus, it is our intent to focus on this design as a case-finding strategy and not as a means to compare IHH with FDR contacts. This also emphasizes the need for good health education with the index case, both in understanding TB symptoms and also the long-term effects of undiagnosed TB. As a result of the small sample size, we could not do meaningful statistical comparisons, and some of our other analyses were potentially influenced by small sample size. However, our selection criteria could save a lot of resources that are very limited in African settings. For this logistical reason, some studies have cautioned against utilizing ACF in low-income settings. ${ }^{35}$ In addition, we did 
not perform spoligotyping, which would have shown whether there was direct transmission of TB between the index case and FDR cases, because it was beyond the scope of the original project and financially impossible. However, the focus of this work was to show that this strategy could be used to find TB cases, regardless of the source case.

\section{Conclusion}

The high TB prevalence among FDR contacts justifies targeting this selected group during any household contact investigation or TB active-case finding. These findings suggest that FDR contacts living in separate households from the index TB case should be considered to be at high risk of Mtb infection and disease and could be efficiently screened among other contacts for Mtb infection and active disease. Our study also demonstrated that it is feasible to initiate ACF among FDR contacts by trained home visitors and to combine this with TB contact investigations among household contacts in low-income setting. This intervention should be part of national TB program strategies to increase the case-detection rate and to reduce TB transmission and death in the community.

\section{Acknowledgments}

We would like to acknowledge the invaluable contribution made by Dr Lorna Nshuti, Dr Christopher Whalen, the study medical officers, health visitors, laboratory, and data personnel: Allan Chiunda, Mark Breda, Dennis Dobbs, Hussein Kisingo, Albert Muganda, Yusuf Mulumba, Deborah Nsamba, Barbara Kyeyune, Faith Kintu, Gladys Mpalanyi, Janet Mukose, Grace Tumusiime, and Philo Nassozi. We would like to acknowledge and thank Dr Francis Adatu Engwau, former Head of the Uganda National Tuberculosis and Leprosy Program, for his support of this project. We would like to acknowledge the medical officers, nurses, and counselors at the National Tuberculosis Treatment Centre, Mulago Hospital, the Ugandan National Tuberculosis and Leprosy Program and the Uganda Tuberculosis Investigation Bacteriological Unit, Wandegeya, for their contributions to this study. This study would not be possible without the generous participation of the Ugandan patients and families.

\section{Funding}

The Kawempe Community Health Study, which forms the basis of this project, was funded entirely by the Tuberculosis Research Unit (grants N01-AI95383 and HHSN266200700022C/N01-AI70022 from the National
Institutes of Health National Institute of Allergy and Infectious Diseases, awarded to WHB). This work was also supported in part by the National Institutes of Health National Heart Lung and Blood Institutes (NHLBI) grant R01HL096811 awarded to CMS.

\section{Disclosure}

The authors report no conflicts of interest in this work.

\section{References}

1. World Health Organization. Global Tuberculosis Report. Geneva, Switzerland: World Health Organization; 2014.

2. World Health Organization. Global Tuberculosis Control: Surveillance, Planning, Financing: WHO Report 2007. Geneva, Switzerland: World Health Organization; 2007.

3. Becerra MC, Pachao-Torreblanca IF, Bayona J, et al. Expanding tuberculosis case detection by screening household contacts. Public Health Rep. 2005;120(3):271-277.

4. World Health Organization. Guidance for National Tuberculosis Programmes on the Management of Tuberculosis in Children. Geneva, Switzerland: World Health Organization; 2006.

5. World Health Organization. Guidelines for Intensified Tuberculosis Case-Finding and Isoniazid Preventive Therapy for People Living with HIV in Resource-Constrained Settings. Geneva, Switzerland: World Health Organization; 2010.

6. World Health Organization. Recommendations for Investigating Contacts of Persons with Infectious Tuberculosis in Low- and MiddleIncome Countries. Geneva, Switzerland: World Health Organization; 2012.

7. Hopewell PC, Pai M, Maher D, Uplekar M, Raviglione MC. International standards for tuberculosis care. Lancet Infect Dis. 2006; 6(11):710-725.

8. Thind D, Charalambous S, Tongman A, Churchyard G, Grant AD. An evaluation of 'Ribolola': a household tuberculosis contact tracing programme in North West Province, South Africa. Int J Tuberc Lung Dis. 2012;16(12):1643-1648.

9. Rieder HL. Contacts of tuberculosis patients in high-incidence countries. Int J Tuberc Lung Dis. 2003;7(12 Suppl 3):S333-S336.

10. Centers for Disease Control and Prevention. Division of Tuberculosis Elimination (DTBE) in Cambodia, Household Contact Investigations, 2010. Available from: http://www.cdc.gov/tb/topic/globaltb/cambodia. htm. Accessed 10 Aug, 2015.

11. Morrison J, Pai M, Hopewell PC. Tuberculosis and latent tuberculosis infection in close contacts of people with pulmonary tuberculosis in low-income and middle-income countries: a systematic review and meta-analysis. Lancet Infect Dis. 2008;8(6):359-368.

12. Akhtar S, Rathi SK. Multilevel modeling of household contextual determinants of tuberculin skin test positivity among contacts of infectious tuberculosis patients, Umerkot, Pakistan. Am J Trop Med Hyg. 2009;80(3):351-358.

13. Guwatudde D, Nakakeeto M, Jones-Lopez EC, et al. Tuberculosis in household contacts of infectious cases in Kampala, Uganda. Am J Epidemiol. 2003;158(9):887-898

14. Whalen CC, Chiunda A, Zalwango S, et al. Immune correlates of acute Mycobacterium tuberculosis infection in household contacts in Kampala, Uganda. Am J Trop Med Hyg. 2006;75(1):55-61.

15. Sia IG, Orillaza RB, St Sauver JL, et al. Tuberculosis attributed to household contacts in the Philippines. Int J Tuberc Lung Dis. 2010;14(1): 122-125.

16. Radhakrishna S, Frieden TR, Subramani R, Santha T, Narayanan PR. Additional risk of developing $\mathrm{TB}$ for household members with a TB case at home at intake: a 15-year study. Int J Tuberc Lung Dis. 2007;11(3): 282-288. 
17. Jackson-Sillah D, Hill PC, Fox A, et al. Screening for tuberculosis among 2381 household contacts of sputum-smear-positive cases in The Gambia. Trans R Soc Trop Med Hyg. 2007;101(6):594-601.

18. Kilicaslan Z, Kiyan E, Kucuk C, et al. Risk of active tuberculosis in adult household contacts of smear-positive pulmonary tuberculosis cases. Int J Tuberc Lung Dis. 2009;13(1):93-98.

19. Jia Z, Cheng S, Ma Y, et al. Tuberculosis burden in China: a high prevalence of pulmonary tuberculosis in household contacts with and without symptoms. BMC Infect Dis. 2014;14:64.

20. Chapman JS, Dyerly MD. Social and other factors in intrafamilial transmission of tuberculosis. Am Rev Respir Dis. 1964;90:48-60.

21. Lienhardt C, Fielding K, Sillah J, et al. Risk factors for tuberculosis infection in sub-Saharan Africa: a contact study in The Gambia. Am J Respir Crit Care Med. 2003;168(4):448-455.

22. Lienhardt C, Sillah J, Fielding K, et al. Risk factors for tuberculosis infection in children in contact with infectious tuberculosis cases in the Gambia, West Africa. Pediatrics. 2003;111(5 pt 1):e608-e614.

23. Moller M, Hoal EG. Current findings, challenges and novel approaches in human genetic susceptibility to tuberculosis. Tuberculosis (Edinb). 2010;90(2):71-83.

24. Stein CM. Genetic epidemiology of tuberculosis susceptibility: impact of study design. PLoS Pathog. 2011;7(1):e1001189.

25. Aissa K, Madhi F, Ronsin N, et al; CG94 Study Group. Evaluation of a model for efficient screening of tuberculosis contact subjects. Am J Respir Crit Care Med. 2008;177(9):1041-1047.

26. Fox GJ, Nhung NV, Sy DN, et al. Contact investigation in households of patients with tuberculosis in Hanoi, Vietnam: a prospective cohort study. PLoS One. 2012;7(11):e49880.

27. Singh J, Sankar MM, Kumar S, et al. Incidence and prevalence of tuberculosis among household contacts of pulmonary tuberculosis patients in a peri-urban population of South Delhi, India. PLoS One. 2013;8(7):e69730.

28. Stein CM, Nshuti L, Chiunda AB, et al. Evidence for a major gene influence on tumor necrosis factor-alpha expression in tuberculosis: path and segregation analysis. Hum Hered. 2005;60(2):109-118.

29. ATS. Diagnostic Standards and Classification of Tuberculosis in Adults and Children. This official statement of the American Thoracic Society and the Centers for Disease Control and Prevention was adopted by the ATS Board of Directors, July 1999. This statement was endorsed by the Council of the Infectious Disease Society of America, September 1999. Am J Respir Crit Care Med. 2000;161(4 pt 1):1376-1395.

30. Wampande EM, Mupere E, Debanne SM, et al. Long-term dominance of Mycobacterium tuberculosis Uganda family in peri-urban Kampala-Uganda is not associated with cavitary disease. BMC Infect Dis. 2013;13:484.
31. Gagneux S, Small PM. Global phylogeography of Mycobacterium tuberculosis and implications for tuberculosis product development. Lancet Infect Dis. 2007;7(5):328-337.

32. de Vries G, van Hest NA, Baars HW, Sebek MM, Richardus JH. Factors associated with the high tuberculosis case rate in an urban area. Int $J$ Tuberc Lung Dis. 2010;14(7):859-865.

33. Guwatudde D, Zalwango S, Kamya MR, et al. Burden of tuberculosis in Kampala, Uganda. Bull World Health Organ. 2003;81(11):799-805.

34. Lienhardt C. From exposure to disease: the role of environmental factors in susceptibility to and development of tuberculosis. Epidemiol Rev. 2001;23(2):288-301.

35. Fox GJ, Barry SE, Britton WJ, Marks GB. Contact investigation for tuberculosis: a systematic review and meta-analysis. Eur Respir J. 2013;41(1):140-156.

36. Sekandi JN, Sempeera H, List J, Whalen C. Missed opportunity for tuberculosis case detection in household contacts in a high burden setting. Pan Afr Med J. 2012;12:8.

37. Sekandi JN, List J, Luzze H, et al. Yield of undetected tuberculosis and human immunodeficiency virus coinfection from active case finding in urban Uganda. Int J Tuberc Lung Dis. 2014;18(1):13-19.

38. Sekandi JN, Neuhauser D, Smyth K, Whalen CC. Active case finding of undetected tuberculosis among chronic coughers in a slum setting in Kampala, Uganda. Int J Tuberc Lung Dis. 2009;13(4):508-513.

39. Blok L, Sahu S, Creswell J, Alba S, Stevens R, Bakker MI Comparative meta-analysis of tuberculosis contact investigation interventions in eleven high burden countries. PLoS One. 2015;10(3): e0119822.

40. Nishikiori N, Van Weezenbeek C. Target prioritization and strategy selection for active case-finding of pulmonary tuberculosis: a tool to support country-level project planning. BMC Public Health. 2013; 13:97.

41. Yadav RP, Nishikiori N, Satha P, Eang MT, Lubell Y. Cost-effectiveness of a tuberculosis active case finding program targeting household and neighborhood contacts in Cambodia. Am J Trop Med Hyg 2014;90(5):866-872.

42. Sekandi JN, Dobbin K, Oloya J, Okwera A, Whalen CC, Corso PS. Costeffectiveness analysis of community active case finding and household contact investigation for tuberculosis case detection in urban Africa. PLoS One. 2015;10(2):e0117009.

43. Graham SM, Ahmed T, Amanullah F, et al. Evaluation of tuberculosis diagnostics in children: 1 . Proposed clinical case definitions for classification of intrathoracic tuberculosis disease. Consensus from an expert panel. J Infect Dis. 2012;205(Suppl 2):S199-S208.
Clinical Epidemiology

\section{Publish your work in this journal}

Clinical Epidemiology is an international, peer-reviewed, open access, online journal focusing on disease and drug epidemiology, identification of risk factors and screening procedures to develop optimal preventative initiatives and programs. Specific topics include: diagnosis, prognosis, treatment, screening, prevention, risk factor modification,

\section{Dovepress}

systematic reviews, risk \& safety of medical interventions, epidemiology \& biostatistical methods, and evaluation of guidelines, translational medicine, health policies \& economic evaluations. The manuscript management system is completely online and includes a very quick and fair peer-review system, which is all easy to use. 